\title{
CONSTRUCTION OF HOMOTOPY EQUIVALENCE OF TRUNCATED COMPLEXES
}

\author{
Yanping Rao and Gang Yang \\ Received: 10 June 2015; Revised: 2 August 2015 \\ Communicated by Abdullah Harmancı
}

\begin{abstract}
For a ring $R$, given two truncated proper left $\mathcal{C}$-resolutions of equal length for the same module, where $\mathcal{C}$ is a subcategory of $R$-modules, we obtain a pair of complexes of the same homotopy type and give some examples.
\end{abstract}

Mathematics Subject Classification (2010): 16E05

Keywords: Proper resolution, homotopy equivalent, precover, precovering class

\section{Introduction}

Throughout this paper, all rings are associative with identity and all modules are unitary modules. Let $R$ be a ring. We denote by $R$-Mod the category of left $R$-modules.

Truncated projective resolutions are of interest in both algebraic geometry and algebraic topology. The final modules of two truncated projective resolutions of the same module may be stabilized to produce homotopy equivalent complexes.

In 2007, Mannan in [3] considered two truncated projective resolutions of equal length for the same module and obtained a pair of complexes of the same homotopy type. This paper generalizes projective resolutions to proper left $\mathcal{C}$-resolutions and similar results are obtained, where $\mathcal{C}$ is a subcategory of $R$-modules. Moreover, some examples are given.

\section{Main results}

Let $\mathcal{C}$ be a subcategory of $R$-modules. Recall that a complex of modules

$$
X=\cdots \rightarrow X_{1} \rightarrow X_{0} \rightarrow X^{0} \rightarrow X^{1} \rightarrow \cdots
$$

in $R$-Mod is called $\operatorname{Hom}_{R}(\mathcal{C},-)$-exact (respectively, $\operatorname{Hom}_{R}(-, \mathcal{C})$-exact) if it remains exact after applying the functor $\operatorname{Hom}_{R}(C,-)$ (respectively, $\operatorname{Hom}_{R}(-, C)$ ) for any object $C \in \mathcal{C}$.

This work was partly supported by NSF of China (Grant No. 11561039), and NSF of Gansu Province of China (No. 145RJZA079). 
Definition 2.1. (see [1]) Let $\mathcal{C}$ be a subcategory of $R$-modules and $M$ a module in $R$-Mod. A homomorphism $f: C \rightarrow M$ with $C \in \mathcal{C}$ is called a $\mathcal{C}$-precover of $M$ if the abelian group homomorphism $\operatorname{Hom}_{R}\left(C^{\prime}, f\right): \operatorname{Hom}_{R}\left(C^{\prime}, C\right) \rightarrow \operatorname{Hom}_{R}\left(C^{\prime}, M\right)$ is surjective for any object $C^{\prime} \in \mathcal{C}$. If every $R$-module has a $\mathcal{C}$-precover, we say that $\mathcal{C}$ is a precovering class. Dually, we have the definitions of a $\mathcal{C}$-preenvelope and a preenveloping class.

Definition 2.2. Let $\mathcal{C}$ be a subcategory of $R$-modules and $M$ a module in $R$-Mod. A complex $\cdots \rightarrow C_{1} \rightarrow C_{0} \rightarrow M \rightarrow 0$ is called a proper left $\mathcal{C}$-resolution of $M$ if each object $C_{i} \in \mathcal{C}$ and if $C_{0} \rightarrow M, C_{i+1} \rightarrow \operatorname{Ker}\left(C_{i} \rightarrow C_{i-1}\right)$ for $i \geq 0$ are all $\mathcal{C}$-precovers (equivalently, the complex $X$ is $\operatorname{Hom}_{R}\left(\mathcal{C},-\right.$-)exact), where $C_{-1}=M$. Dually, a proper right $\mathcal{C}$-resolution $0 \rightarrow M \rightarrow C^{0} \rightarrow C^{1} \rightarrow \cdots$ of $M$ can be defined. A proper $\mathcal{C}$-resolution of $M$ is a complex $\cdots \rightarrow C_{1} \rightarrow C_{0} \rightarrow C^{0} \rightarrow C^{1} \rightarrow \cdots$, where $M=\operatorname{Im}\left(C_{0} \longrightarrow C^{0}\right)$, complex $\cdots \rightarrow C_{1} \rightarrow C_{0} \rightarrow M \rightarrow 0$ is a proper left $\mathcal{C}$-resolution of $M$ and complex $0 \rightarrow M \rightarrow C^{0} \rightarrow C^{1} \rightarrow \cdots$ is a proper right $\mathcal{C}$ resolution of $M$.

Let

$$
X=\cdots \longrightarrow X_{i+1} \stackrel{\alpha_{i+1}}{\longrightarrow} X_{i} \stackrel{\alpha_{i}}{\longrightarrow} X_{i-1} \stackrel{\alpha_{i-1}}{\longrightarrow} \cdots
$$

and

$$
Y=\cdots \longrightarrow Y_{i+1} \stackrel{\beta_{i+1}}{\longrightarrow} Y_{i} \stackrel{\beta_{i}}{\longrightarrow} Y_{i-1} \stackrel{\beta_{i-1}}{\longrightarrow} \cdots
$$

be two complexes. Recall that a chain map $f: X \rightarrow Y$ is a family of morphisms $\left\{f_{i}\right\}$ such that the following diagram commutes:

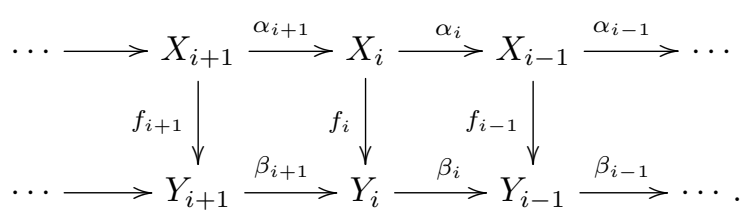

Recall that two chain maps $f, g: X \rightarrow Y$ are said to be chain homotopic, denoted by $f \sim g$, if there exists a family of morphisms $\left\{s_{i}\right\}$ with each $s_{i}: X_{i} \rightarrow Y_{i+1}$ a morphism such that

$$
f_{i}-g_{i}=\beta_{i+1} s_{i}+s_{i-1} \alpha_{i} .
$$

The complexes $X$ and $Y$ are said to be chain homotopy equivalent, if there exist chain maps $f: X \rightarrow Y$ and $g: Y \rightarrow X$, such that $f g \sim I_{Y}$ and $g f \sim I_{X}$, where $I_{Y}, I_{X}$ are identity maps.

Lemma 2.3. Let $F=\cdots \rightarrow F_{1} \rightarrow F_{0} \rightarrow F^{0} \rightarrow F^{1} \rightarrow \cdots$ be a $\operatorname{Hom}_{R}(\mathcal{C},-)$ exact sequence of $R$-modules. By adding $\cdots \rightarrow 0 \rightarrow E \stackrel{i d}{\rightarrow} E \rightarrow 0 \rightarrow \cdots$ to $F$, 
then the sequence $G=\cdots \rightarrow F_{1} \rightarrow F_{0} \oplus E \rightarrow F^{0} \oplus E \rightarrow F^{1} \rightarrow \cdots$ is $\operatorname{Hom}_{R}(\mathcal{C},-)$ exact. Furthermore, the sequences $F$ and $G$ are chain homotopy equivalent.

Proof. For any $C \in \mathcal{C}$, applying the functor $\operatorname{Hom}_{R}(C,-)$ to $G$, we have the following commutative diagram

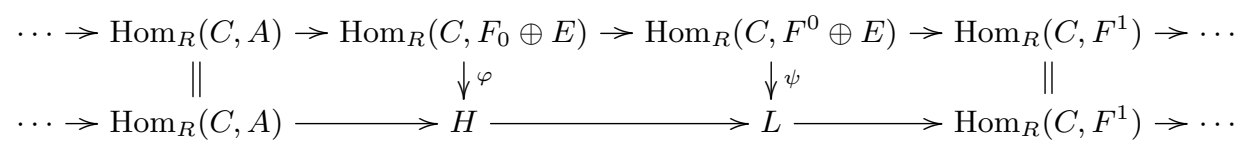

where $H=\operatorname{Hom}_{R}\left(C, F_{0}\right) \oplus \operatorname{Hom}_{R}(C, E), L=\operatorname{Hom}_{R}\left(C, F^{0}\right) \oplus \operatorname{Hom}_{R}(C, E), \varphi$ and $\psi$ are isomorphisms. It is easy to see that the lower sequence in the above diagram is exact, so is the upper sequence. Thus the sequence $G$ is $\operatorname{Hom}_{R}(\mathcal{C},-)$-exact. That sequences $F$ and $G$ are chain homotopy equivalent is simple. This completes the proof.

The following result plays a crucial role in this paper.

Theorem 2.4. Let $\mathcal{C}$ be a subcategory of $R$-modules such that $\mathcal{C}$ is closed under finite direct sums, and $M$ a module in R-Mod. Suppose that we have two proper left $\mathcal{C}$-resolutions of $M$ :

$$
C_{n} \stackrel{\alpha_{n}}{\longrightarrow} C_{n-1} \stackrel{\alpha_{n-1}}{\longrightarrow} \cdots \stackrel{\alpha_{2}}{\longrightarrow} C_{1} \stackrel{\alpha_{1}}{\longrightarrow} C_{0} \stackrel{\sigma}{\longrightarrow} M \longrightarrow 0
$$

and

$$
C_{n}^{\prime} \stackrel{\alpha_{n}^{\prime}}{\longrightarrow} C_{n-1}^{\prime} \stackrel{\alpha_{n-1}^{\prime}}{\longrightarrow} \cdots \stackrel{\alpha_{2}^{\prime}}{\longrightarrow} C_{1}^{\prime} \stackrel{\alpha_{1}^{\prime}}{\longrightarrow} C_{0}^{\prime} \stackrel{\sigma^{\prime}}{\longrightarrow} M \longrightarrow 0 .
$$

Then the complexes

$$
C_{n} \oplus S_{n} \stackrel{\alpha_{n} \oplus 0}{\longrightarrow} C_{n-1} \stackrel{\alpha_{n-1}}{\longrightarrow} \cdots \stackrel{\alpha_{2}}{\longrightarrow} C_{1} \stackrel{\alpha_{1}}{\longrightarrow} C_{0}
$$

and

$$
C_{n}^{\prime} \oplus T_{n} \stackrel{\alpha_{n}^{\prime} \oplus 0}{\longrightarrow} C_{n-1}^{\prime} \stackrel{\alpha_{n-1}^{\prime}}{\longrightarrow} \cdots \stackrel{\alpha_{2}^{\prime}}{\longrightarrow} C_{1}^{\prime} \stackrel{\alpha_{1}^{\prime}}{\longrightarrow} C_{0}^{\prime}
$$

are chain homotopy equivalent, where the modules $T_{i}, S_{i}$ are defined inductively by $T_{0} \cong C_{0}, S_{0} \cong C_{0}^{\prime}$, and for $i=1,2, \cdots, n, T_{i} \cong C_{i} \oplus S_{i-1}, S_{i} \cong C_{i}^{\prime} \oplus T_{i-1}$.

Proof. We follow the proof of [3, Theorem 1.1]. For each $i=1,2, \cdots, n$, we have natural inclusions of summands: $\lambda_{i}: C_{i} \rightarrow T_{i}, \lambda_{i}^{\prime}: C_{i}^{\prime} \rightarrow S_{i}$. Let $\lambda_{0}: C_{0} \rightarrow T_{0}$ and $\lambda_{0}: C_{0}^{\prime} \rightarrow S_{0}$ both be the identity maps. We define $\rho_{i}: T_{i} \rightarrow T_{i-1} \oplus S_{i-1}$, and $\rho_{i}^{\prime}: S_{i} \rightarrow S_{i-1} \oplus T_{i-1}$ by

$$
\rho_{i}=\left(\begin{array}{cc}
\lambda_{i-1} \alpha_{i} & 0 \\
0 & 1
\end{array}\right) \quad \rho_{i}^{\prime}=\left(\begin{array}{cc}
\lambda_{i-1}^{\prime} \alpha_{i}^{\prime} & 0 \\
0 & 1
\end{array}\right) .
$$


For $r=0,1, \cdots, n-1$, let $\mathcal{C}_{r}$ denote the chain complex

$$
C_{n} \oplus S_{n} \stackrel{\alpha_{n} \oplus 0}{\longrightarrow} \cdots \stackrel{\alpha_{r+2}}{\longrightarrow} C_{r+1} \stackrel{\lambda_{r} \alpha_{r+1}}{\longrightarrow} T_{r} \stackrel{\rho_{r}}{\longrightarrow} T_{r-1} \oplus S_{r-1} \stackrel{\rho_{r-1} \oplus 0}{\longrightarrow} \cdots \stackrel{\rho_{1} \oplus 0}{\longrightarrow} T_{0} \oplus S_{0} .
$$

Also let $\mathcal{C}_{n}$ denote chain complex

$$
T_{n} \oplus S_{n} \stackrel{\rho_{n} \oplus 0}{\longrightarrow} T_{n-1} \oplus S_{n-1} \stackrel{\rho_{n-1} \oplus 0}{\longrightarrow} \cdots \stackrel{\rho_{2} \oplus 0}{\longrightarrow} T_{1} \oplus S_{1} \stackrel{\rho_{1} \oplus 0}{\longrightarrow} T_{0} \oplus S_{0} .
$$

Clearly, $\mathcal{C}_{0}$ is the chain complex $\left(\natural^{\prime}\right)$. For $r=0,1, \cdots, n-1$, the chain complex $\mathcal{C}_{r+1}$ is obtained from $\mathcal{C}_{r}$ by replacing $\stackrel{\alpha_{r+2}}{\longrightarrow} C_{r+1} \stackrel{\lambda_{r} \alpha_{r+1}}{\longrightarrow} T_{r} \stackrel{\delta_{r}}{\longrightarrow}$ with

$$
\stackrel{\lambda_{r+1} \alpha_{r+2}}{\longrightarrow} C_{r+1} \oplus S_{r} \stackrel{\rho_{r+1}}{\longrightarrow} T_{r} \oplus S_{r} \stackrel{\rho_{r} \oplus 0}{\longrightarrow} .
$$

Similarly, for $r=0,1, \cdots, n-1$, let $\mathcal{D}_{r}$ denote the chain complex

$$
C_{n}^{\prime} \oplus T_{n} \stackrel{\alpha_{n}^{\prime} \oplus 0}{\longrightarrow} \cdots \stackrel{\alpha_{r+2}^{\prime}}{\longrightarrow} C_{r+1}^{\prime} \stackrel{\lambda_{r}^{\prime} \alpha_{r+1}^{\prime}}{\longrightarrow} S_{r} \stackrel{\rho_{r}^{\prime}}{\longrightarrow} S_{r-1} \oplus T_{r-1} \stackrel{\rho_{r-1}^{\prime} \oplus 0}{\longrightarrow} \cdots \stackrel{\rho_{1}^{\prime} \oplus 0}{\longrightarrow} S_{0} \oplus T_{0}
$$

Again let $\mathcal{D}_{n}$ denote chain complex

$$
S_{n} \oplus T_{n} \stackrel{\rho_{n}^{\prime} \oplus 0}{\longrightarrow} S_{n-1} \oplus T_{n-1} \stackrel{\rho_{n-1}^{\prime} \oplus 0}{\longrightarrow} \cdots \stackrel{\rho_{2}^{\prime} \oplus 0}{\longrightarrow} S_{1} \oplus T_{1} \stackrel{\rho_{1}^{\prime} \oplus 0}{\longrightarrow} S_{0} \oplus T_{0} .
$$

Clearly, $\mathcal{D}_{0}$ is the chain complex $\left(\sharp^{\prime}\right)$.

By Lemma 2.3, for $i=0,1, \cdots, n, \mathcal{C}_{i}$ and $\mathcal{D}_{i}$ are $\operatorname{Hom}_{R}(\mathcal{C},-)$-exact. For $r=0,1, \cdots, n-1, \mathcal{C}_{r+1}$ (respectively, $\mathcal{D}_{r+1}$ ) is chain homotopy equivalent to $\mathcal{C}_{r}$ (respectively, $\mathcal{D}_{r}$ ). Hence $\left(\natural^{\prime}\right)$ (respectively, $\left(\sharp^{\prime}\right)$ ) is chain homotopy equivalent to $\mathcal{C}_{n}$ (respectively, $\mathcal{D}_{n}$ ).

To prove that $\left(\natural^{\prime}\right)$ and $\left(\sharp^{\prime}\right)$ are chain homotopy equivalent, it suffices to show that $\mathcal{C}_{n}$ is chain isomorphic to $\mathcal{D}_{n}$, that is, there exist isomorphisms $h_{i}, k_{i}$ making the following diagram commute:

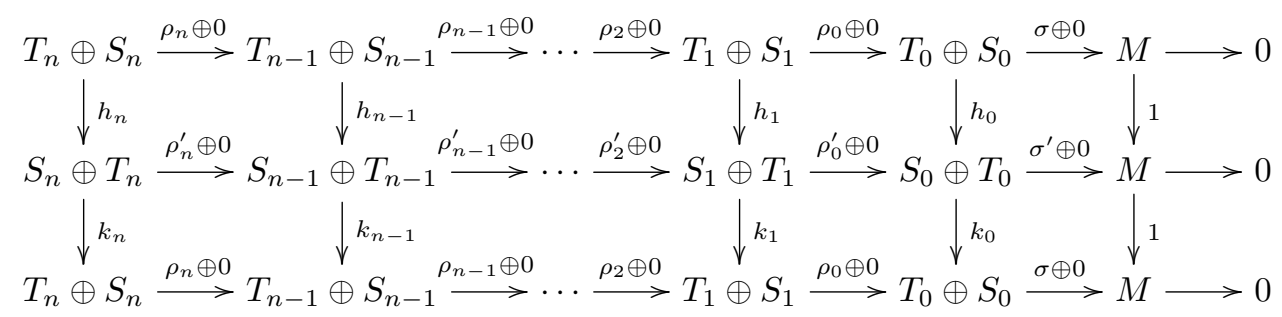

For $i=0,1, \cdots, n, T_{i}, S_{i} \in \mathcal{C}$ since $\mathcal{C}$ is closed under finite direct sums.

We proceed by induction on $n$. For $n=0$, as the sequences $T_{0} \stackrel{\sigma}{\rightarrow} M \rightarrow 0$ and $S_{0} \stackrel{\sigma^{\prime}}{\rightarrow} M \rightarrow 0$ are $\operatorname{Hom}_{R}(\mathcal{C},-)$-exact, there exist $f_{0}, g_{0}$ such that the following 
diagrams commute:
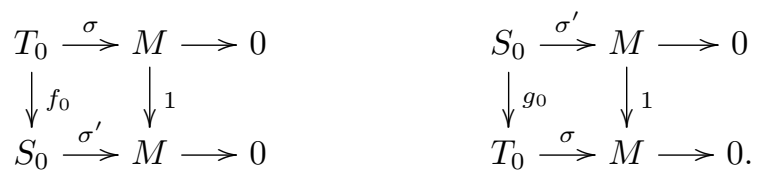

(b)

Define $h_{0}: T_{0} \oplus S_{0} \rightarrow S_{0} \oplus T_{0}$ and $k_{0}: S_{0} \oplus T_{0} \rightarrow T_{0} \oplus S_{0}$ by

$$
h_{0}=\left(\begin{array}{cc}
f_{0} & 1-f_{0} g_{0} \\
1 & -g_{0}
\end{array}\right), k_{0}=\left(\begin{array}{cc}
g_{0} & 1-g_{0} f_{0} \\
1 & -f_{0}
\end{array}\right) .
$$

Then $h_{0} k_{0}=1$ and $k_{0} h_{0}=1$. From commutativity of $(b)$, we deduce:

$$
\left(\sigma^{\prime}, 0\right) h_{0}=(\sigma, 0),(\sigma, 0) k_{0}=\left(\sigma^{\prime}, 0\right)
$$

Hence we get the following commutative diagrams:
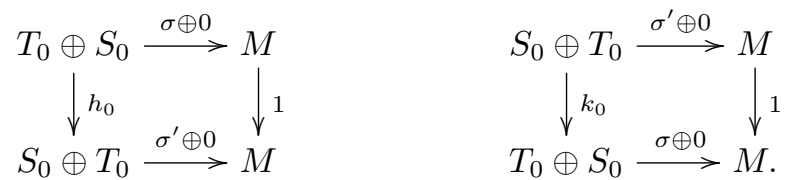

Now suppose that for some $j<i \leq n$, we have defined $h_{j}: T_{j} \oplus S_{j} \rightarrow S_{j} \oplus T_{j}$ and $k_{j}: S_{j} \oplus T_{j} \rightarrow T_{j} \oplus S_{j}$ for $j=0,1, \cdots, i-1$, so that for each $j$, we have $h_{j} k_{j}=1$ and $k_{j} h_{j}=1$.

Since the sequences $T_{i} \stackrel{\rho_{i}}{\rightarrow} \operatorname{Ker}\left(\rho_{i-1} \oplus 0\right) \quad$ and $\quad S_{i} \stackrel{\rho_{i}^{\prime}}{\rightarrow} \operatorname{Ker}\left(\rho_{i-1}^{\prime} \oplus 0\right)$ are $\operatorname{Hom}_{R}(\mathcal{C},-)$-exact, and $T_{i}, S_{i} \in \mathcal{C}$, there exist $f_{i}, g_{i}$ such that the following diagrams commute:

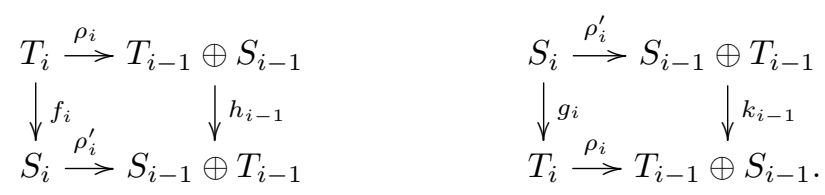

Define $h_{i}: T_{i} \oplus S_{i} \rightarrow S_{i} \oplus T_{i}$ and $k_{i}: S_{i} \oplus T_{i} \rightarrow T_{i} \oplus S_{i}$ by

$$
h_{i}=\left(\begin{array}{cc}
f_{i} & 1-f_{i} g_{i} \\
1 & -g_{i}
\end{array}\right) \quad k_{i}=\left(\begin{array}{cc}
g_{i} & 1-g_{i} f_{i} \\
1 & -f_{i}
\end{array}\right) .
$$

Then $h_{i} k_{i}=1$ and $k_{i} h_{i}=1$. Recall $h_{i-1} k_{i-1}=1$ and $k_{i-1} h_{i-1}=1$. From commutativity of $\left(b^{\prime}\right)$, we deduce:

$$
\left(\rho_{i}^{\prime}, 0\right) h_{i}=h_{i-1}\left(\rho_{i}, 0\right),\left(\rho_{i}, 0\right) k_{i}=k_{i-1}\left(\rho_{i}^{\prime}, 0\right) .
$$


Hence we get the following commutative diagrams:
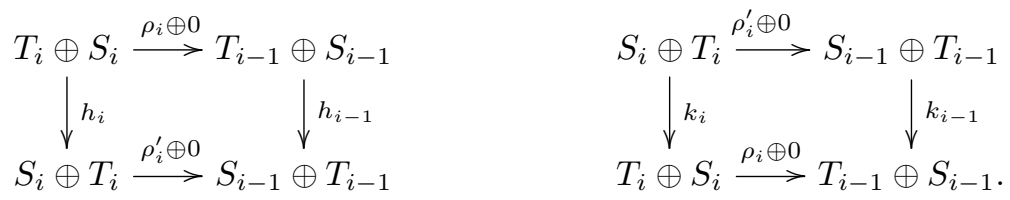

So $\mathcal{C}_{n}$ is chain isomorphic to $\mathcal{D}_{n},\left(\mathfrak{\natural}^{\prime}\right)$ and $\left(\sharp^{\prime}\right)$ are chain homotopy equivalent. This completes the proof.

As applications of Theorem 2.4, we will give some examples. Firstly, the following result follows immediately from Theorem 2.4 since the class of projective modules is closed under direct sums.

Corollary 2.5. ([3, Theorem 1.1]) Let $R$ be a ring and $M$ a module in $R$-Mod. Suppose we have exact sequences:

$$
P_{n} \stackrel{\partial_{n}}{\longrightarrow} P_{n-1} \stackrel{\partial_{n-1}}{\longrightarrow} \cdots \stackrel{\partial_{2}}{\longrightarrow} P_{1} \stackrel{\partial_{1}}{\longrightarrow} P_{0} \stackrel{\epsilon}{\longrightarrow} M \longrightarrow 0
$$

and

$$
Q_{n} \stackrel{\partial_{n}^{\prime}}{\longrightarrow} Q_{n-1} \stackrel{\partial_{n-1}^{\prime}}{\longrightarrow} \cdots \stackrel{\partial_{2}^{\prime}}{\longrightarrow} Q_{1} \stackrel{\partial_{1}^{\prime}}{\longrightarrow} Q_{0} \stackrel{\epsilon^{\prime}}{\longrightarrow} M \longrightarrow 0
$$

with the $P_{i}$ and $Q_{i}$ all projective modules in $R$-Mod. Then the complexes

$$
P_{n} \oplus S_{n} \stackrel{\partial_{n} \oplus 0}{\longrightarrow} P_{n-1} \stackrel{\partial_{n-1}}{\longrightarrow} \cdots \stackrel{\partial_{2}}{\longrightarrow} P_{1} \stackrel{\partial_{1}}{\longrightarrow} P_{0}
$$

and

$$
Q_{n} \oplus T_{n} \stackrel{\partial_{n}^{\prime} \oplus 0}{\longrightarrow} Q_{n-1} \stackrel{\partial_{n-1}^{\prime}}{\longrightarrow} \cdots \stackrel{\partial_{2}^{\prime}}{\longrightarrow} Q_{1} \stackrel{\partial_{1}^{\prime}}{\longrightarrow} Q_{0}
$$

are chain homotopy equivalent, where the modules $T_{i}, S_{i}$ are defined inductively by $T_{0} \cong P_{0}, S_{0} \cong Q_{0}$, and for $i=1,2, \cdots, n, T_{i} \cong P_{i} \oplus S_{i-1}, S_{i} \cong Q_{i} \oplus T_{i-1}$.

Recall from [5] that a module $M$ is called FP-injective if $\operatorname{Ext}_{R}^{1}(F, M)=0$ for any finitely presented module $F$. Recently, Pinzon in [4] shows that every module in $R$-Mod has an FP-injective cover if $R$ is a left coherent ring. So every module $M$ in $R$-Mod has a proper left FP-injective resolution if $R$ is coherent.

Example 2.6. Let $R$ be a left coherent ring and $M$ a module in $R$-Mod. Suppose the complexes ( () ) and $(\sharp)$ in Theorem 2.4 are two proper left FP-injective resolutions of $M$, then the complexes $\left(\mathfrak{\natural}^{\prime}\right)$ and $\left(\sharp^{\prime}\right)$ in Theorem 2.4 are chain homotopy equivalent.

Proof. Note that the class of FP-injective modules in $R$-Mod is closed under direct sums. Then the result follows from Theorem 2.4. 
In the following, we denote the class of all projective, flat and injective $R$ modules, respectively, by $\mathcal{P}(R), \mathcal{F}(R)$ and $\mathcal{I}(R)$.

Definition 2.7. (see [2]) An $R$-module $M$ is called Gorenstein projective if there exists an exact sequence of projective modules $P=\cdots \rightarrow P_{1} \rightarrow P_{0} \rightarrow P^{0} \rightarrow P^{1} \rightarrow \cdots$ such that $M \cong \operatorname{Im}\left(P_{0} \rightarrow P^{0}\right)$ and $P$ is $\operatorname{Hom}_{R}(-, \mathcal{P}(R))$-exact. In this case, we say $P$ is a complete projective resolution of $M$. Gorenstein injective modules are defined dually.

Holm in [2] shows that, every module $M$ in $R$-Mod with finite Gorenstein dimension admits a proper left Gorenstein projective resolution.

Example 2.8. Let $M$ be a module in R-Mod. Suppose the complexes ( $\square)$ and $(\sharp)$ in Theorem 2.4 are two proper left Gorenstein projective resolutions of $M$, then the complexes $\left(\natural^{\prime}\right)$ and $\left(\sharp^{\prime}\right)$ in Theorem 2.4 are chain homotopy equivalent.

Proof. Note that the class of Gorenstein projective modules in $R$-Mod is closed under finite direct sums. Then the result follows from Theorem 2.4.

Example 2.9. Let $M$ be a Gorenstein injective module. Then $M$ admits a proper left $\mathcal{I}(R)$-resolution by the dual of $[2$, Proposion 2.3]. Suppose the complexes ( $\square$ ) and $(\sharp)$ in Theorem 2.4 are two proper left $\mathcal{I}(R)$-resolutions of $M$, then the complexes $\left(\mathfrak{\natural}^{\prime}\right)$ and $\left(\sharp^{\prime}\right)$ in Theorem 2.4 are chain homotopy equivalent.

Theorem 2.10. Let $\mathcal{C}$ be a subcategory of $R$-modules such that $\mathcal{C}$ is closed under finite direct sums and $M$ a module in $R$-Mod. Suppose we have two proper right $\mathcal{C}$-resolutions of $M$ :

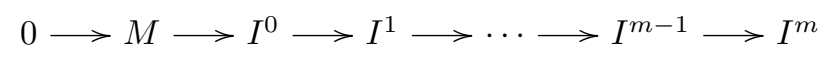

and

$$
0 \longrightarrow M \longrightarrow J^{0} \longrightarrow J^{1} \longrightarrow \cdots \longrightarrow J^{m-1} \longrightarrow J^{m} .
$$

Then the complexes

$$
I^{0} \longrightarrow I^{1} \longrightarrow \cdots \longrightarrow I^{m-1} \longrightarrow I^{m} \oplus S^{m}
$$

and

$$
J^{0} \longrightarrow J^{1} \longrightarrow \cdots \longrightarrow J^{m-1} \longrightarrow J^{m} \oplus T^{m}
$$

are chain homotopy equivalent, where the modules $T^{i}, S^{i}$ are defined inductively by $T^{0} \cong I^{0}, S^{0} \cong J^{0}$, and for $i=1,2, \cdots, m, T^{i} \cong I^{i} \oplus S^{i-1}, S^{i} \cong J^{i} \oplus T^{i-1}$.

Proof. The proof is dual to that of Theorem 2.4. 
It was showed in [1, Proposion 6.5.1.] that, a ring $R$ is right coherent if and only if the class of flat left modules in $R$-Mod is preenveloping. So every module $M$ in $R$-Mod has a proper right flat resolution if $R$ is coherent.

Example 2.11. Let $R$ be a right coherent ring and $M$ a module in $R$-Mod. Suppose the complexes $(\dagger)$ and $(\ddagger)$ in Theorem 2.10 are two proper right $\mathcal{F}(R)$-resolutions of $M$. Then the complexes $\left(\dagger^{\prime}\right)$ and $\left(\ddagger^{\prime}\right)$ in Theorem 2.10 are chain homotopy equivalent.

Example 2.12. Let $M$ be a Gorenstein projective module. Then $M$ admits a proper right $\mathcal{P}(R)$-resolution by $[2$, Proposion 2.3]. Suppose the complexes $(\dagger)$ and $(\ddagger)$ in Theorem 2.10 are two proper right $\mathcal{P}(R)$-resolutions of $M$, then the complexes $\left(\dagger^{\prime}\right)$ and $\left(\ddagger^{\prime}\right)$ in Theorem 2.10 are chain homotopy equivalent.

Proposition 2.13. Let $\mathcal{C}$ be a subcategory of $R$-modules such that $\mathcal{C}$ is closed under finite direct sums and $M$ a module in R-Mod. Suppose we have two proper $\mathcal{C}$-resolutions of $M$ :

$$
P_{n} \rightarrow P_{n-1} \rightarrow \cdots \rightarrow P_{1} \rightarrow P_{0} \rightarrow P^{0} \rightarrow P^{1} \rightarrow \cdots \rightarrow P^{m-1} \rightarrow P^{m}
$$

and

$$
Q_{n} \rightarrow Q_{n-1} \rightarrow \cdots \rightarrow Q_{1} \rightarrow Q_{0} \rightarrow Q^{0} \rightarrow Q^{1} \rightarrow \cdots \rightarrow Q^{m-1} \rightarrow Q^{m} .
$$

Then the complexes

$$
P_{n} \oplus S_{n} \stackrel{\partial_{n}}{\rightarrow} P_{n-1} \stackrel{\partial_{n}-1}{\rightarrow} \cdots \stackrel{\partial_{2}}{\rightarrow} P_{1} \stackrel{\partial_{1}}{\rightarrow} P_{0} \stackrel{\partial_{0}}{\rightarrow} P^{0} \stackrel{\partial^{0}}{\rightarrow} P^{1} \stackrel{\partial^{1}}{\rightarrow} \cdots \stackrel{\partial^{m-2}}{\rightarrow} P^{m-1} \stackrel{\partial^{m-1}}{\rightarrow} P^{m} \oplus S^{m}
$$

and

$$
Q_{n} \oplus T_{n} \rightarrow Q_{n-1} \rightarrow \cdots \rightarrow Q_{1} \rightarrow Q_{0} \rightarrow Q^{0} \rightarrow Q^{1} \rightarrow \cdots \rightarrow Q^{m-1} \rightarrow Q^{m} \oplus T^{m}
$$

are chain homotopy equivalent, where the modules $T_{i}, S_{i}, T^{i}, S^{i}$ are defined inductively by $T_{0} \cong P_{0}, S_{0} \cong Q_{0}, T^{0} \cong P^{0}, S^{0} \cong Q^{0}$ and for $i=1,2, \cdots, n$, $T_{i} \cong P_{i} \oplus S_{i-1}, S_{i} \cong Q_{i} \oplus T_{i-1}, T^{i} \cong P^{i} \oplus S^{i-1}, S^{i} \cong Q^{i} \oplus T^{i-1}$.

Proof. For $0 \leq i \leq n$, there exist $f_{i}: P_{i} \rightarrow Q_{i}, g_{i}: Q_{i} \rightarrow P_{i}$ and $s_{i}: P_{i} \rightarrow P_{i+1}$ such that $g_{i+1} f_{i+1}-I_{P_{i+1}}=\partial_{i+2} s_{i+1}+s_{i} \partial_{i+1}$ by Theorem 2.4. For $0 \leq i \leq m$, there exist $f^{i}: P^{i} \rightarrow Q^{i}, g^{i}: Q^{i} \rightarrow P^{i}$ and $s^{i+1}: P^{i+1} \rightarrow P^{i}$ such that $g^{i+1} f^{i+1}-I^{P_{i+1}}=$ $\partial_{i} s_{i+1}+s_{i+2} \partial_{i+1}$ by Theorem 2.10. Let $s^{0}: P^{0} \rightarrow P_{0}$ and $s^{0}=0$. Clearly, we have $g_{0} f_{0}-I_{P_{0}}=\partial_{1} s_{0}+s^{0} \partial_{0}$ and $g^{0} f^{0}-I^{P_{0}}=\partial_{0} s^{0}+s^{1} \partial^{0}$. Thus the complexes (3) and (4) are chain homotopy equivalent. 
Proposition 2.14. Let $M$ be a Gorenstein projective R-module. Suppose the complexes (1) and (2) are two complete projective resolutions of $M$, then the complexes (3) and (4) are chain homotopy equivalent, where the modules $T_{i}, S_{i}, T^{i}, S^{i}$ are defined as in Proposition 2.13 for $i=0,1, \cdots, n$.

Proof. By [3, Theorem 1.1] and Example 2.12.

Acknowledgment. The authors would like to thank the referee for the valuable suggestions and comments.

\section{References}

[1] E. E. Enochs and O. M. G. Jenda, Relative Homological Algebra, De Gruyter Expositions in Mathematics, 30, Walter De Gruyter, Berlin, 2000.

[2] H. Holm, Gorenstein homological dimensions, J. Pure Appl. Algebra, 189(1-3) (2004), 167-193.

[3] W. H. Mannan, Homotopy types of truncated projective resolutions, Homology, Homotopy Appl., 9(2) (2007), 445-449.

[4] K. Pinzon, Absolutely pure covers, Comm. Algebra, 36(6) (2008), 2186-2194.

[5] B. Stenström, Coherent rings and FP-injective modules, J. London Math. Soc., 2(2) (1970), 323-329.

Yanping Rao and Gang Yang

Department of Mathematics

Lanzhou Jiaotong University

Lanzhou 730070, China

e-mails: yprao0213@gmail.com (Y. Rao)

yanggang10@gmail.com (G. Yang) 